\title{
Energy Equilibration Processes of Electrons, Magnons, and Phonons at the Femtosecond Time Scale
}

\author{
J. Walowski, ${ }^{1}$ G. Müller, ${ }^{1}$ M. Djordjevic, ${ }^{1}$ M. Münzenberg, ${ }^{1, *}$ M. Kläui, ${ }^{2}$ C. A. F. Vaz, ${ }^{3, *}$ and J. A. C. Bland ${ }^{3, *}$ \\ 'IV. Physikalisches Institut, Universität Göttingen, Germany, \\ ${ }^{2}$ Fachbereich Physik \& Zukunftskolleg, Universität Konstanz, Germany \\ ${ }^{3}$ Cavendish Laboratory, University of Cambridge, Cambridge, CB3 OHE, United Kingdom
}

\begin{abstract}
We relate the energy dissipation processes at the femtosecond (electron-spin relaxation time $\tau_{\mathrm{el}-\mathrm{sp}}$ ) and nanosecond time scale (Gilbert relaxation $\tau_{\alpha}$ ) to the microscopic model proposed by Koopmans [Phys. Rev. Lett. 95, 267207 (2005)]. At both time scales, Elliot-Yafet scattering is proposed as the dominant contribution. We controllably manipulate the energy dissipation by transition metal doping $(\mathrm{Pd})$ and rare earth doping (Dy) of a Permalloy film. While a change in $\tau_{\alpha}$ of more than a factor of 2 is observed, $\tau_{\mathrm{el}}$-sp remains constant. We explain the discrepancies as due to relaxation channels not considered in the model.
\end{abstract}

A detailed understanding of the excitation mechanisms in ferromagnetic films is a key requirement for predicting temporal limits of specific magnetic switching of a spinelectronic device. The excitation mechanism can be achieved using a number of methods: magnetic field pulses [1-3], relativistic electron bunches [4], resonant excitation in microwave fields $[5,6]$, spin-torque transfer [7], anisotropy field pulses [8,9] and the inverse Faraday effect [10]. However, a complete microscopic understanding of the "magnetic viscosity" given by the Gilbert relaxation $\tau_{a}$ $[11,12]$, critical for engineering the magnetization dynamics involving ultrafast magnetic processes in future magnetic devices, is still lacking. In particular, the role of the spin-orbit coupling connecting the electron system with the magnetic excitations is still unclear: One way it enters is by significantly changing the band structure for spin-up and spin-down states, resulting in Elliot-Yafet scattering [1315]. Based on that mechanism, a microscopic model was recently developed by Koopmans et al. [16] (Koopmans model, for short). The model describes the processes occurring in the fs range by an extended three-temperature model [17], artificially separating electrons, spins, and the lattice, as the system relaxes to equilibrium. Alternative ideas include a band narrowing in the final state recently observed in fs x-ray spectroscopy [18], increasing the energy scale of the spin-orbit interaction, or a quenching of the exchange interaction [19], leading to a "loose spin" model where the energy barrier for a spin flip is significantly reduced at hot electron temperatures. In the Koopmans model, the effects of band-structure variations are neglected. The altered electronic occupancy and the resulting electronic relaxation processes alone already result in an ultrafast demagnetization. An experimental test of the model is therefore necessary to develop a better understanding of the microscopic processes and to test the limits of validity.

In this Letter, the relation between microscopic $\left(\tau_{\mathrm{el}-\mathrm{sp}}\right)$ and the macroscopic variables $\left(\alpha, T_{C}\right)$ predicted by the
Koopmans model

$$
\tau_{\mathrm{el}-\mathrm{sp}} \propto \frac{\hbar}{k_{B} T_{C}} \frac{1}{\alpha}
$$

is studied, where $T_{C}$ is the Curie temperature. We take advantage of fs-pump-probe techniques, which can measure concurrently the relevant energy dissipation processes that occur at both, fs time scale (localized spin-flip) and coherent precession in the $100 \mathrm{ps}$ range (spin-wave vector $k=0$ ) [13]. On the fs time scale, the electron-spin relaxation time $\tau_{\mathrm{el}-\mathrm{sp}}$ determines the demagnetization, while the uniform precession of the magnetization (Kittel mode) can be used to determine the Gilbert damping $\alpha$ [all-optical ferromagnetic resonance (AO-FMR)] [8,9,20,21]. To manipulate the dissipation processes we start with a permalloy (Py, $\mathrm{Fe}_{80} \mathrm{Ni}_{20}$ ) film. The damping is then increased artificially by doping the Py with different concentrations of two different materials classes that increase the induced spin-flip scattering rate $\lambda_{\mathrm{sp}-i}$ [16,22]: $2 \% \mathrm{Pd}$ as a transition metal and $1 \%$ Dy and $2 \%$ Dy as a rare earth to make a comparative study between them.

The ferromagnetic films were deposited on a Si substrate using molecular beam epitaxy (MBE) by coevaporation of the Py and a doping material (Pd, Dy). The thickness of the deposited film was set to $12 \mathrm{~nm}$ (growth rates of $-0.002 \mathrm{~nm} / \mathrm{s}$ ). All films are capped with a $2 \mathrm{~nm} \mathrm{Au}$ film for protection against oxidation. The distribution of the doping within the films was characterized by Rutherford backscattering (RBS) and the average doping is found to be slightly below the nominal value. The doping levels were deliberately chosen to be low. Only for low doping levels is the dopant influence on the band structure of the host Py film expected to be minor and the crystal structure of the Py film to be little disturbed to allow for a comparison with the undoped case [23]. Large variations in $T_{C}$ are not expected in the low doping range where the exchange interaction is dominated by the Py matrix, and are not evidenced in our data [24]. 
In Fig. 1(a) the schematic experimental fs-pump-probe experiment and in (b) the experimental data are shown. The exciting pump pulse uses a fluence of $40 \mathrm{~mJ} / \mathrm{cm}^{2}$ per pulse (80 fs, $\lambda=800 \mathrm{~nm}$ ). The Kerr rotation $\theta_{K}(\tau)$ is measured by a probe pulse delayed by a time $\tau$ after excitation
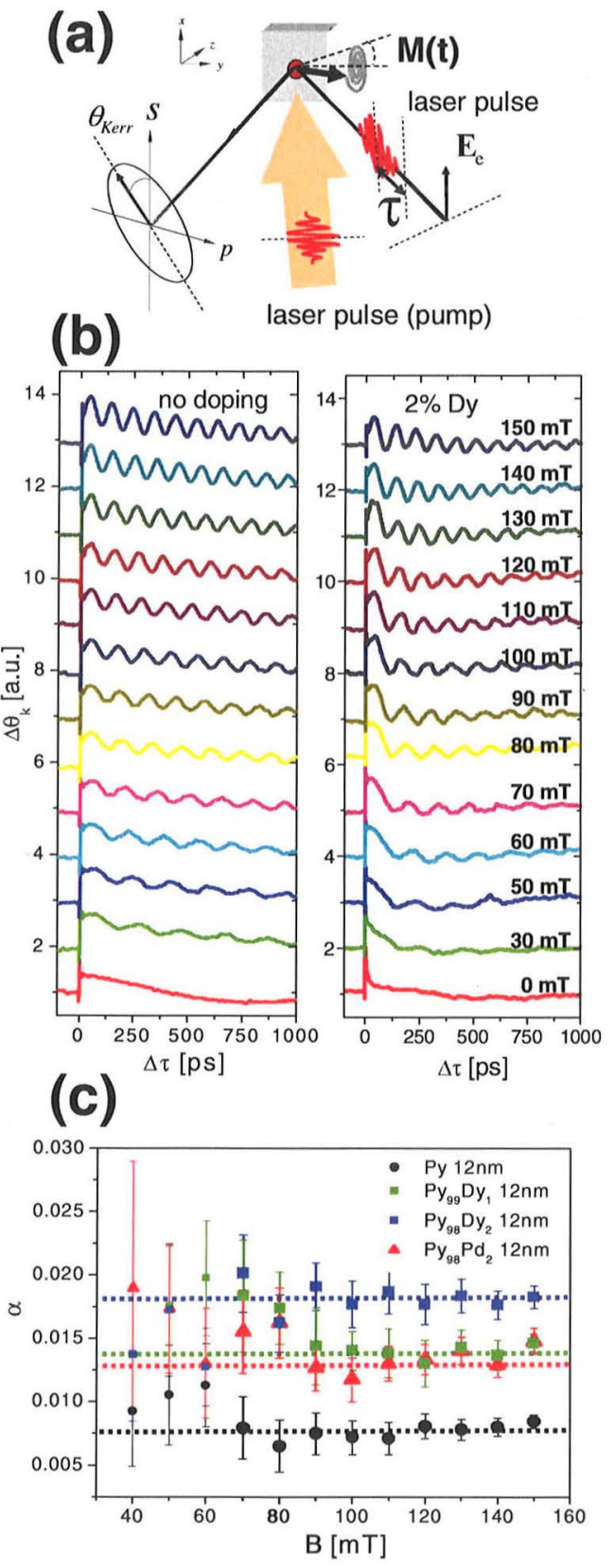

FIG. 1 (color online). (a) Schematic of the pump-probe setup. (b) Experimental data for the undoped permalloy (Py) film and with $2 \%$ Dy doping for different field values (applied $35^{\circ}$ out-ofplane). (c) The extracted values for the Gilbert damping as a function of the applied field showing an increase with Pd and Dy doping. (double modulation technique [25]). A static field is applied $35^{\circ}$ out of plane to generate a ps anisotropy field pulse large enough to start a coherent precession [20,21]. Two data sets corresponding to the $2 \%$ Dy and the undoped Py films are shown in Fig. 1(b). The applied field $H_{\text {ext }}$ is varied from $0-150 \mathrm{mT}$. The data are fitted to a harmonic function with an exponentially decreasing amplitude in order to extract the period of the precession and the decay time $\tau_{\alpha}$ corresponding to the Gilbert damping. A detailed study of the field dependence (Kittel equation) is necessary to determine the parameters of the film $[21,24]$. The Gilbert damping $\alpha$, the decay time $\tau_{\alpha}$, the effective magnetic anisotropy for the thin film $K_{z}$ and the gyromagnetic ratio $\gamma$ are related by [20]

$$
\alpha=\frac{1}{\tau_{\alpha} \gamma}\left(H_{\mathrm{ext}} \cos \phi-\frac{K_{z}}{\mu_{0} M_{s}}+\frac{M_{s}}{2}\right)^{-1}
$$

Within the standard ansatz given by the Landau-LifshitzGilbert (LLG) equation the energy dissipation rate $\alpha$ is assumed to be field independent. In practice, various dissipation processes can contribute [22,26-30]. For the undoped $12 \mathrm{~nm}$ thick Py film, a Gilbert damping $\alpha$ of 0.008 is found. The values obtained for the Gilbert damping are given in Fig. 1(c) for different field values, showing a systematic increase of the Gilbert damping $\alpha$ with increasing doping. For a Dy doping of $1 \%(2 \%)$, the Gilbert damping increases to $0.015(0.02)$. Within the field range from 60 to $150 \mathrm{mT}$, no dependence on $H_{\mathrm{ext}}$ is observed [Fig. 1(c)]. However, in the low field region, fewer oscillations and a reduced amplitude (intrinsic to the excitation mechanism) entail larger error bars. For the same doping level Dy leads to a stronger damping than Pd doping, as expected [31-33]. While for the doping with the $4 d$ transition metal, the interaction between the damping mechanisms originates from $\mathrm{Py} 4 s$-Pd $4 d$ interaction, similar to that in a $s-d$ model $[22,34]$, for the rare earth the energy is expected to be transferred to the Dy $4 f$ system by a Py $3 d$-Dy $4 f$ interaction mediated via the Dy $5 d$ electrons.

The most important microscopic processes in the Koopmans model are spin flips occurring in an electronscattering event. Because of spin-orbit interaction, the spin variable for a single electron does not commute with the Hamiltonian of the system, and the electron bands cannot be separated into "purely" spin-up and -down. The degree of coupling is given by the strength of the spin-orbit interaction. This means that the electron spin is not conserved in a scattering event at the so-called hot spots [15]. If one imagines an electron moving in a band, neglecting spin-orbit coupling as in a standard band-structure calculation, in a scattering process the electron is scattered into a new state, possibly transferring some energy and momentum to the lattice [Fig. 2(a)]. However, in a band structure with some hot spots, with spin-orbit interaction turned on [Fig. 2(b)], if the electron is scattered into a state that is partly spin up and down at a so-called hot-spot, the spin 


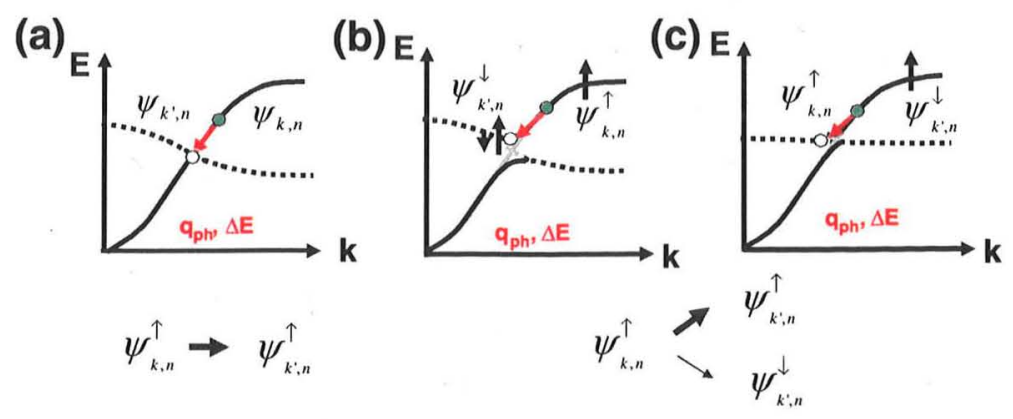

FIG. 2 (color online). Processes leading to spin flips in an electron-scattering event in a simplified schematic band picture. (a) The bands are not spin split and the spin-orbit coupling is neglected. (b) When spin-orbit interaction is included, the bands cannot be separated into purely spin-up and purely spin-down. By scattering at so-called hot spots where bands are strongly intermixed, there is a high probability that the electron will have its spin flipped after the scattering event. (c) Rare earth $4 f$ bands are very localized and thus only weakly hybridized.

will flip with some probability on that same time scale. This mechanism directly connects the electron-lattice relaxation with the electron-spin relaxation.

In Fig. 3, the ultrafast (fs time scale) demagnetization data are shown for all samples [35]. The demagnetization process happens very quickly within the first 100-200 fs and, in contrast to the Koopmans model, is not significantly changed by the Dy and Pd doping. To determine accurately the relaxation times, the slopes of the curves are analyzed using the analytic solution for the three-temperature model given by Dalla Longa et al. [36]. The two exponential functions mirror the demagnetization given by $\tau_{\mathrm{cl}-\text { sp }}$ and the decay of the electron temperature $\tau_{\text {el-lat }}$ owing to the transfer of energy to the lattice:

$$
\begin{aligned}
\Delta M= & \theta(t)\left(-A_{1}+\frac{A_{2} \tau_{\text {el-lat }}-A_{1} \tau_{\text {el-sp }}}{\tau_{\text {cl-lat }}-\tau_{\text {el-sp }}} e^{-\left(t / \tau_{\mathrm{el}-\text { sp }}\right)}\right. \\
& \left.+\frac{A_{1}-A_{2}}{\tau_{\text {el-lat }}-\tau_{\text {el-sp }}} \tau_{\text {el-lat }} e^{-\left(t / \tau_{\text {el-lat }}\right)}\right) M_{0}{ }^{* t} \Gamma(t),
\end{aligned}
$$

$\theta(t)$ is the step function and ${ }^{*} \Gamma(t)$ stands for a convolution with the laser pulse envelope determining the temporal resolution. A plot of the demagnetization times $\tau_{\text {el-sp }}$ against the Gilbert damping $\alpha$ is shown in Fig. 3, inset. Theoretically, a strong decrease of $\tau_{\text {el-sp }}$ with $\alpha$ is expected. For comparison, the functional dependence of $\tau_{\text {el-sp }}$ vs $\alpha$ is shown according to (1) for $T_{C}$ and $0.9 T_{C}$, respectively. We find that the demagnetization times do not follow the predicted decrease with increasing damping.

At first glance these findings seem to provide a falsification of the model and these results are supported by similar findings for Py doped with different rare earth materials [33]. In general, there are different kinds of energy dissipation processes contributing to the Gilbert damping $\alpha$ even if one assumes only intrinsic electronic energy dissipation channels. Since the Py films are MBEgrown and have been prepared by coevaporation with very low evaporation rates under ultrahigh vacuum (base pres- sure $10^{-10}$ mbar) conditions, we expect a low extrinsic contribution [33]. At a closer look, the deduction of (1) is only valid for electronic energy dissipation channels that appear at the short time scale in the same way as on longer time scales. This is unlikely to be the case for Dy doping: The rare earth $4 f$ bands are almost atomiclike and narrow in energy far above or below the Fermi energy owing slight hybridization [Fig. 2(c)]. Therefore, they will not contribute as an ultrafast electronic relaxation channel at short

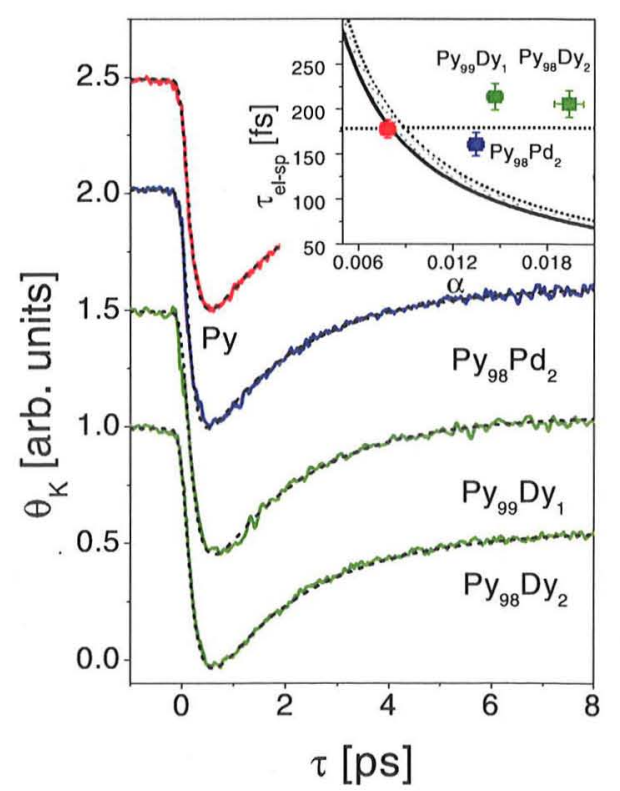

FIG. 3 (color online). The ultrafast demagnetization for $12 \mathrm{~nm}$ thick permalloy (Py) films, undoped and with $\mathrm{Pd}(2 \%)$ and Dy $(1 \%, 2 \%)$ doping. To extract the demagnetization time, $\tau_{\mathrm{el}-\mathrm{sp}}$ is analyzed with an analytic solution of the three-temperature model. In the inset the demagnetization time is plotted as a function of the Gilbert damping $\alpha$ for $T_{C}$ and $0.9 T_{C}$, where $T_{C}$ is the Py Curie temperature. The horizontal line marks the value for the undoped Py film as a reference. 
time scales. Nevertheless, on the long time scale, a slow energy dissipation channel arises from a transfer of the precessional motion to the rare earth by distortion of the lattice and a repopulation of the lowest $4 f$ levels with a temporal lag due to the rare earth ion relaxation time [37]. It is proposed to be responsible for the increased Gilbert damping $\alpha$ in rare earth doped Py films [33]. In contrast, Pd states with their $5 d$ and $6 s$ states will be much broader in energy and more hybridized and so the Koopmans model should be more valid. For this case, we find a tendency for the demagnetization time $\tau_{\mathrm{el}-\mathrm{sp}}$ to decrease with the increased Gilbert damping, even though the effect is found to be smaller than theoretically predicted. While it is clear that the model is not applicable for rare earth doping, we suggest that future investigations focus on the damping induced by transition metals, where the predictions of the Koopmans model are followed more closely, at least qualitatively.

In summary, we show that we are able to tune the microscopic energy dissipation process in Py by low dose doping with a $4 d$ transition metal (Pd) and a rare earth (Dy) material. Using fs all-optical pump-probe experiments both the ultrafast demagnetization $\left(\tau_{\mathrm{el}-\mathrm{sp}}\right)$ and magnetic damping $\left(\tau_{\alpha}\right)$ were ascertained simultaneously. While the Koopmans model predicts that an increase in the magnetic energy dissipation results in a faster demagnetization time, this is clearly not observed in the Dy-doped permalloy. The different electronic nature of the rare earth dopant suggests a different coupling between electrons, magnons, and phonons on the femtosecond time scale. For Pd doping, only a qualitative agreement is found. We explain the failure of the model in terms of missing electronic relaxation channels for the case of the rare earth doping.

Support by the Deutsche Forschungsgemeinschaft within the priority program SPP 1133 is gratefully acknowledged. This work was partly supported by the EPSRC (UK). We thank B. Koopmans, D. Steiauf, G. Woltersdorf, and I. Radu for discussion and for sharing their results prior to publication.

\section{*Corresponding author} mmuenze@gwdg.de

Present address: Applied Physics, Yale University, New Haven, CT 06520, USA.

Deceased.

[1] B. C. Choi, J. Ho, G. Arnup, and M.R. Freeman, Phys. Rev. Lett. 95, 237211 (2005).

[2] B. Van Waeyenberge et al., Nature (London) 444, 461 (2006).

[3] M. Buess et al., Phys. Rev. Lett. 93, 077207 (2004).

[4] I. Tudosa et al., Nature (London) 428, 831 (2004).

[5] S. Tamaru et al., J. Appl. Phys. 91, 8034 (2002).

[6] A. Puzic et al., J. Appl. Phys. 97, 10E704 (2005).

[7] M. Tsoi et al., Nature (London) 406, 46 (2000); E. B. Myers et al., Science 285, 867 (1999).
[8] M. van Kampen et al., Phys. Rev. Lett. 88, 227201 (2002).

[9] G. Ju et al., Phys. Rev. B 57, R700 (1998).

[10] C. D. Stanciu et al., Phys. Rev. Lett. 98, 207401 (2007).

[11] T. L. Gilbert, IEEE Trans. Magn. 40, 3443 (2004).

[12] M. Fähnle et al., Phys. Rev. B 73, 172408 (2006).

[13] M. Djordjevic and M. Münzenberg, Phys. Rev. B 75, 012404 (2007).

[14] R.J. Elliott, Phys. Rev. 96, 266 (1954); Y. Yafet, Solid State Phys. 14, 1 (1963).

[15] J. Fabian and S. Das Sarma, Phys. Rev. Lett. 81, 5624 (1998); J. Appl. Phys. 85, 5075 (1999).

[16] B. Koopmans et al., Phys. Rev. Lett. 95, 267207 (2005).

[17] E. Beaurepaire et al., Phys. Rev. Lett. 76, 4250 (1996).

[18] C. Stamm et al., Nature Mater. 6, 740 (2007).

[19] H.-S. Rhie, H. A. Dürr, and W. Eberhardt, Phys. Rev. Lett. 90, 247201 (2003).

[20] M. Djordjevic, Ph. D. thesis, University of Göttingen, 2006.

[21] M. Djordjevic et al., J. Appl. Phys. 99, 08F308 (2006); J. Walowski et al., J. Phys. D 41, 164016 (2008).

[22] V. Kamberský, Can. J. Phys. 48, 2906 (1970).

[23] M. Finazzi et al., Phys. Rev. Lett. 75, 4654 (1995).

[24] The room temperature values $g=2.12$ and $\mu_{0} M_{S}=$ $0.8 \mathrm{~T}$ for Py are used. $K_{z}$ is determined as a fit parameter: the absolute value increases with Dy doping from $K_{Z}=$ -1.5 to $-4.7 \times 10^{4} \mathrm{~J} / \mathrm{m}^{3}$ at maximum of $2 \%$ Dy doping for the thin film (corresponding to a $0.5 \mathrm{GHz}$ shift to higher frequencies). See EPAPS Document No. EPRLTAO-101-022847 for supplementary figures and details. For more information on EPAPS, see http://www. aip.org/pubservs/epaps.html.

[25] B. Koopmans in Spin Dynamics in Confined Magnetic Structures II, Topics in Applied Physics, edited by B. Hillebrands and K. Ounadjela (Springer-Verlag, Berlin, 2003), Vol. 87.

[26] G. Eilers et al., Phys. Rev. B 74, 054411 (2006).

[27] R. Arias and D. L. Mills, Phys. Rev. B 60, 7395 (1999).

[28] G. Woltersdorf and B. Heinrich, Phys. Rev. B 69, 184417 (2004)

[29] J. Lindner et al., Phys. Rev. B 68, 060102 (2003).

[30] J. O. Rantschler and C. Alexander, J. Appl. Phys. 93, 6665 (2003).

[31] S. G. Reidy, L. Cheng, and W. E. Bailey, Appl. Phys. Lett. 82, 1254 (2003).

[32] J. O. Rantschler et al., J. Appl. Phys. 101, 033911 (2007).

[33] G. Woltersdorf et al., arXiv:0802.3206v2; I. Radu (private communications).

[34] A. H. Mitchell, Phys. Rev. 105, 1439 (1957).

[35] We consider here the asymmetric part of the Kerr rotation, $\theta_{K,-}(\tau)=\frac{1}{2}\left(\theta_{K}(\tau, M)-\theta_{K}(\tau,-M)\right)$. The transient reflectivity $R(\tau)$ mirrors the electron dynamics, revealing a faster saturation not altered by the doping. See EPAPS Document No. E-PRLTAO-101-022847 for supplementary figures and details. For more information on EPAPS, see http://www.aip.org/pubservs/epaps.html.

[36] F. Dalla Longa, J. T. Kohlhepp, W. J. M. de Jonge, and B. Koopmans, Phys. Rev. B 75, 224431 (2007).

[37] J.H. Van Vleck and R. Orbach, Phys. Rev. Lett. 11, 65 (1963). 